\title{
Particulate matter exposures and adult-onset asthma and COPD in the Nurses' Health Study
}

\begin{abstract}
To the Editor:
Exposure to ambient air pollution has been associated with acute respiratory effects, including acute exacerbations of asthma and chronic obstructive pulmonary disease (COPD) [1, 2]. However, evidence on the association of long-term exposure to air pollution and incidence of asthma or COPD is limited, with most studies focused on asthma in children [3]. Among adults, recent reviews for both asthma [3] and COPD [4] discuss several studies that demonstrate either no increased risk or a suggestion of risk associated with traffic-related pollutants. With few exceptions [5-7], most have not used robust exposure assessments, large cohorts or fine-particulate exposures. Using previously validated spatiotemporal exposure models [8], we examined the association between long-term exposure to particulate matter (PM) air pollution and incident cases of adult-onset asthma and COPD from 1992 to 2000 in the US Nurses' Health Study (NHS).

The NHS is a prospective cohort of 121701 female nurses who were between 30 and 55 years of age at the start of follow-up in 1976. Participants complete mailed questionnaires every 2 years on a multitude of risk factors and health outcomes, including asthma, emphysema and chronic bronchitis. Response rates are $>90 \%$ for each follow-up cycle. We defined cases as those reporting a physician diagnosis of asthma or COPD on biennial questionnaires, and who subsequently reported use of an asthma medication within the past 12 months or reported a diagnostic test at the time of COPD diagnosis on supplemental questionnaires (1998 and 2000). These case definitions have been previously validated for both asthma [9] and COPD [10]. Time-varying ambient exposure estimates at each participant's residence to $\mathrm{PM} \leqslant 2.5 \mu \mathrm{m}(\mathrm{PM} 2.5), \leqslant 10 \mu \mathrm{m}$ (PM10), and between 2.5 and $10 \mu \mathrm{m}$ in aerodynamic diameter (PM10-2.5) were assessed using nationwide spatiotemporal models that incorporated data from multiple pollution monitor networks and various geospatial predictors [8]. Model predictions of exposure were available monthly from January 1988. We also examined the effect of residential distance to primary or secondary roads, as a proxy for traffic-related exposures, in separate regression models. Participants were divided into three categories (0-50, 50-200 and $\geqslant 200 \mathrm{~m}$ ) corresponding to the closest distance of their residence to an A1, A2 or A3 road [11].
\end{abstract}

Follow-up began in 1992 to allow for calculation of 4-year moving average PM exposures and continued through 2000, the date of the last supplemental questionnaire. Cox proportional hazards models were used to examine whether asthma or COPD was associated with 4-year average exposure to PM2.5, PM10 or PM10-2.5. Basic models were adjusted for region, and stratified by age and calendar year. Fully adjusted models included potential confounders selected a priori based on the previous literature, and included age, time period, geographic region, body mass index, alcohol consumption, physical activity, census-tract median household income, Western dietary pattern [12], second-hand smoke exposure at home and work, smoking status, and pack-years smoked. Stratified models were used to examine effect modification by smoking status. Sensitivity analyses restricted to nonmovers or to participants residing within metropolitan statistical areas (MSAs) were conducted. To examine the effect of varying exposure averaging times, separate models were run with 2-year, 3-year or total cumulative (since 1988) moving average PM exposures. We excluded prevalent cases of asthma or COPD at baseline, as well as participants missing exposure or year of diagnosis.

There were 934 incident cases of asthma among 104254 participants during 796208 person-years of follow-up and 372 incident cases of COPD among 103838 participants during 795964 person-years of follow-up. Incident asthma cases were primarily among never-smokers $(43 \%, \mathrm{n}=400)$ and former smokers $(45 \%, \mathrm{n}=417)$, while the majority of COPD cases were among current smokers $(58 \%, \mathrm{n}=214)$ and former smokers $(30 \%$, $\mathrm{n}=111)$. Mean \pm SD PM exposures over the follow-up period were $24.0 \pm 6.3,14.2 \pm 3.2$ and $9.8 \pm 4.4 \mu \mathrm{g} \cdot \mathrm{m}^{-3}$ for PM10, PM2.5, and PM10-2.5, respectively. The average age during follow-up was $61.5 \pm 7.4$ years.

In this cohort, there was no consistent evidence of an association between exposure to PM and incident asthma or COPD (table 1). In fully adjusted models, the hazard ratios (HRs) for a $10-\mu \mathrm{g} \cdot \mathrm{m}^{-3}$ increase in 4-year average PM10, PM2.5 or PM10-2.5 were 0.94 (95\% CI 0.84-1.06), 0.90 (95\% CI 0.73-1.12) and 0.93 (95\% CI $0.77-1.13$ ) for asthma, and 0.91 (95\% CI 0.76-1.10), 0.93 (95\% CI 0.66-1.31) and 0.83 (95\% CI 0.60-1.14) for COPD, respectively. There was a suggestion of higher risk for COPD across all size fractions of PM among never-smokers, though no statistically significant associations were observed among this subpopulation. In addition, no statistically significant associations were observed for residential proximity to roads with incident asthma or COPD. In sensitivity analyses, we found comparable results after limiting 
TABLE 1 Hazard ratios and $95 \%$ confidence intervals of the association of incident asthma and chronic obstructive pulmonary disease (COPD) with 4-year cumulative average particulate matter (PM) exposures ${ }^{\#}$ or residential proximity to road categories? in the Nurses' Health Study

\begin{tabular}{|c|c|c|c|c|c|c|c|c|}
\hline & \multicolumn{2}{|c|}{ Full cohort } & \multicolumn{2}{|c|}{ Never-smokers } & \multicolumn{2}{|c|}{ Former smokers } & \multicolumn{2}{|c|}{ Current smokers } \\
\hline & Basic $^{+}$ & Adjusted $^{\S}$ & Basic $^{+}$ & Adjusted $^{\S}$ & Basic $^{+}$ & Adjusted $^{\S}$ & Basic $^{+}$ & Adjusted $^{\S}$ \\
\hline \multicolumn{9}{|l|}{ Asthma } \\
\hline \multicolumn{9}{|c|}{ Modelled PM fraction } \\
\hline $\mathrm{PM}_{10}$ & $0.92(0.82-1.03)$ & $0.94(0.84-1.06)$ & $0.97(0.82-1.15)$ & $0.98(0.83-1.17)$ & $0.95(0.80-1.13)$ & $0.96(0.80-1.14)$ & $0.64(0.46-0.91)$ & $0.66(0.47-0.94)$ \\
\hline $\mathrm{PM} 2.5$ & $0.87(0.70-1.08)$ & $0.90(0.73-1.12)$ & $0.88(0.64-1.21)$ & $0.90(0.65-1.23)$ & $0.97(0.70-1.34)$ & $0.98(0.71-1.36)$ & $0.55(0.29-1.02)$ & $0.57(0.30-1.07)$ \\
\hline $\mathrm{PM} 10-2.5$ & $0.89(0.73-1.08)$ & $0.93(0.77-1.13)$ & $1.02(0.77-1.35)$ & $1.04(0.79-1.38)$ & $0.89(0.66-1.20)$ & $0.90(0.67-1.21)$ & $0.48(0.26-0.88)$ & $0.50(0.27-0.92)$ \\
\hline \multicolumn{9}{|c|}{ Distance to $\operatorname{road}^{f} \mathrm{~m}$} \\
\hline $50-199$ & $0.91(0.77-1.07)$ & $0.90(0.77-1.07)$ & $0.96(0.74-1.25)$ & $0.96(0.74-1.25)$ & $0.85(0.66-1.09)$ & $0.84(0.66-1.08)$ & $0.98(0.61-1.56)$ & $0.93(0.58-1.51)$ \\
\hline $0-49$ & $1.15(0.94-1.40)$ & $1.13(0.93-1.38)$ & $1.21(0.89-1.65)$ & $1.20(0.88-1.64)$ & $1.18(0.89-1.57)$ & $1.14(0.85-1.51)$ & $1.02(0.55-1.89)$ & $1.04(0.56-1.94)$ \\
\hline \multicolumn{9}{|l|}{ COPD } \\
\hline \multicolumn{9}{|c|}{ Modelled PM fraction } \\
\hline $\mathrm{PM} 10$ & $0.92(0.76-1.11)$ & $0.91(0.76-1.10)$ & $1.12(0.69-1.81)$ & $1.14(0.70-1.87)$ & $0.91(0.64-1.28)$ & $0.90(0.64-1.27)$ & $0.83(0.63-1.08)$ & $0.87(0.67-1.13)$ \\
\hline $\mathrm{PM} 2.5$ & $0.87(0.61-1.23)$ & $0.93(0.66-1.31)$ & $1.17(0.47-2.90)$ & $1.23(0.50-3.06)$ & $0.94(0.50-1.78)$ & $1.00(0.53-1.88)$ & $0.72(0.45-1.15)$ & $0.82(0.51-1.31)$ \\
\hline $\mathrm{PM} 10-2.5$ & $0.88(0.64-1.21)$ & $0.83(0.60-1.14)$ & $1.19(0.54-2.65)$ & $1.22(0.54-2.76)$ & $0.80(0.44-1.44)$ & $0.74(0.41-1.34)$ & $0.78(0.50-1.22)$ & $0.80(0.51-1.25)$ \\
\hline \multicolumn{9}{|c|}{ Distance to road $^{f} \mathrm{~m}$} \\
\hline $50-199$ & $1.07(0.83-1.38)$ & $0.98(0.76-1.27)$ & $1.22(0.60-2.45)$ & $1.28(0.63-2.59)$ & $1.05(0.66-1.68)$ & $0.93(0.57-1.49)$ & $0.96(0.68-1.35)$ & $0.91(0.64-1.29)$ \\
\hline $0-49$ & $1.05(0.76-1.45)$ & $0.96(0.69-1.32)$ & $0.65(0.22-1.92)$ & $0.67(0.23-1.98)$ & $1.07(0.60-1.93)$ & $1.04(0.58-1.90)$ & $1.04(0.68-1.57)$ & $1.01(0.66-1.55)$ \\
\hline
\end{tabular}

PM10: particulate matter $\leqslant 10 \mu \mathrm{m}$ in aerodynamic diameter; PM2.5: particulate matter $\leqslant 2.5 \mu \mathrm{m}$ in aerodynamic diameter; PM10-2.5: particulate matter between 2.5 and $10 \mu \mathrm{m}$ in aerodynamic diameter. \#: models are for $1992-2000$ and for $10 \mu \mathrm{g} \cdot \mathrm{m}^{-3}$ increase in 4 -year cumulative average particulate matter exposures; there were 104254 participants 1796 208 person-years, 934 cases) included in the asthma analysis and 103838 participants (795964 person-years, 372 cases) included in the COPD analysis. ": models are for $1992-2000$ and use distance of $\geqslant 200 \mathrm{~m}$ as the reference group; there were 81231 participants (620818 person-years, 767 cases) in the asthma analysis and 82616 participants (633 138 person-years, 306 cases) in the COPD analysis. ${ }^{+}$: models adjusted for age, time period, and geographic region. ${ }^{\S}$ : additionally adjusted for body mass index, alcohol consumption, physical activity, census-tract median household income, Western dietary pattern [12], second-hand smoke exposure at home and work, smoking status (when not stratified by status), and pack-years. : included A1-A3 categories as defined by US Census Bureau [11]; A1, primary roads, typically interstate highways, with limited access, division between the opposing directions of traffic and defined exits; A2, primary major, noninterstate highways and major roads without access restrictions; A3, smaller, secondary roads, usually with more than two lanes. 
to nonmovers or MSA residents and results were consistent in separate models using different exposure averaging times (table 2).

There are few previous studies examining the association between exposure to PM and adult-onset asthma to which we can compare our results. In their analysis of the Sister Study, a US cohort of predominantly non-Hispanic white women, Young et al. [5] found a suggestion of increased risk of adult-onset asthma with PM2.5 (HR 1.20, 95\% CI 0.99-1.46 per $3.6 \mu \mathrm{g} \cdot \mathrm{m}^{-3}$ ). Additionally, a recent meta-analysis of six cohorts participating in the European Studies on Chronic Air Pollution Effects (ESCAPE) project found nonsignificant positive associations with PM10 (OR 1.04, 95\% CI 0.88-1.23 per $10 \mu \mathrm{g} \cdot \mathrm{m}^{-3}$ ) and PM2.5 (OR 1.04, 95\% CI $0.88-1.23$ per $5 \mu \mathrm{g} \cdot \mathrm{m}^{-3}$ ), and a nonsignificant negative association with PM10-2.5 (OR 0.98, 95\% CI 0.87-1.14 per $5 \mu \mathrm{g} \cdot \mathrm{m}^{-3}$ ) [7]. No evidence of modification by smoking status was found in stratified analysis [7].

Our results are similar to those from other studies of long-term exposure to PM and incidence of COPD. A study of four cohorts participating in the ESCAPE project found no association with PM and incident COPD; however, traffic intensity on the nearest major road was positively associated with incident COPD in females and never-smokers [6].

Many of the current hypotheses on the pathophysiological mechanisms linking ambient air pollution to incident asthma or COPD focus on the role of pollution-induced oxidative stress and free radical reactions that may lead to airway damage and inflammation $[13,14]$. These concepts could help explain our findings of lower risk of asthma and COPD among current smokers, as cigarette smoke may saturate the same pathways [15] and minimise any additional adverse effect from ambient particles. However, it should be noted that the relatively few incident cases of asthma among current smokers and COPD among never-smokers limited our assessment of risk in these subgroups.

Although this study used advanced and validated spatiotemporal models to estimate PM exposure, some exposure misclassification was unavoidable due to date of diagnosis being limited to self-reported year of first physician diagnosis on the supplemental questionnaires. Thus, our exposure estimates for cases may have been separated from the month of self-reported diagnosis by up to 6 months. In response, we chose a longer (4 years) exposure averaging metric and considered other averaging times in sensitivity analyses.

TABLE 2 Hazard ratios and 95\% confidence intervals for incident asthma/chronic obstructive pulmonary disease (COPD) with 4-year moving average particulate matter (PM) exposures among all, metropolitan statistical area (MSA)-restricted ${ }^{\#}$ and nonmoving" participants of the Nurses' Health Study ${ }^{+}$with 2-, 3- and 4-year, and cumulative average particulate matter exposures ${ }^{\S}$

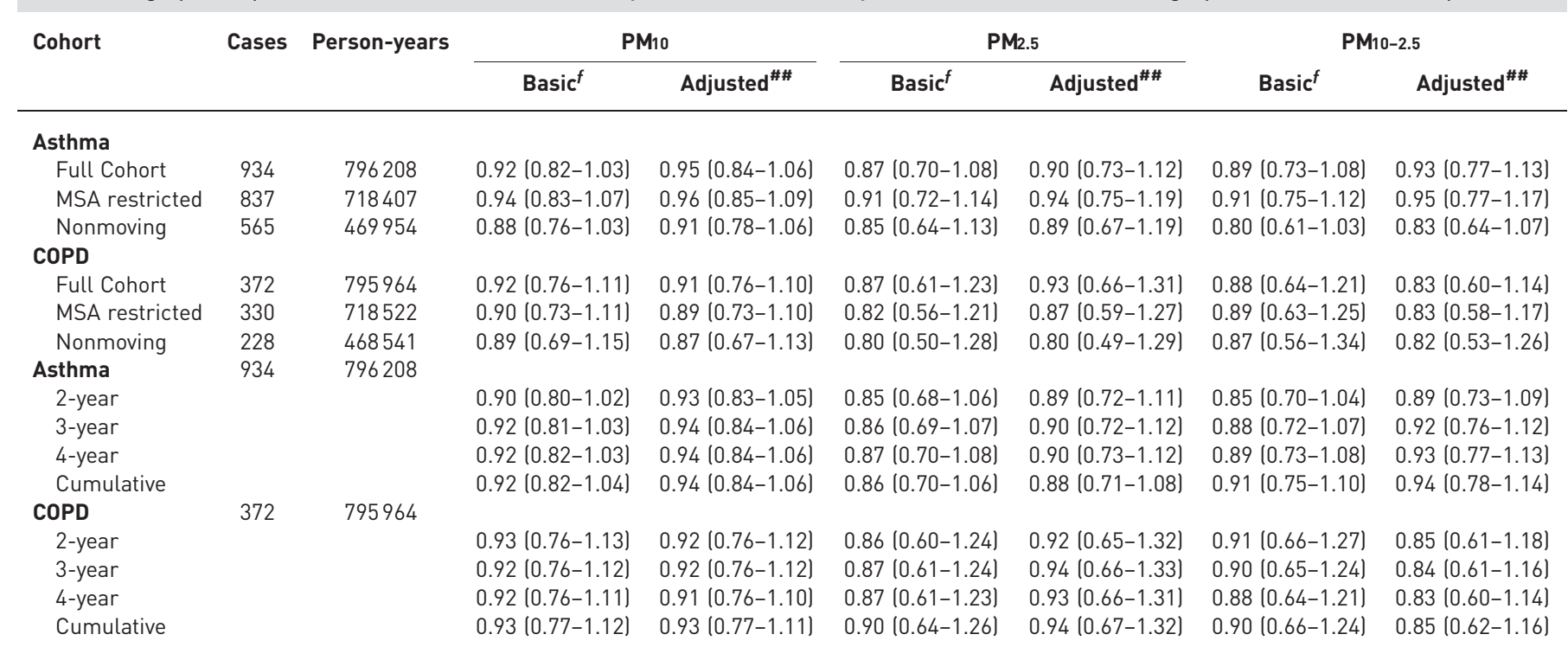

PM10: particulate matter $\leqslant 10 \mu \mathrm{m}$ in aerodynamic diameter; PM2.5: particulate matter $\leqslant 2.5 \mu \mathrm{m}$ in aerodynamic diameter; PM10-2.5: particulate matter between 2.5 and $10 \mu \mathrm{m}$ in aerodynamic diameter. "\#: limited to participants who resided in a Metropolitan Statistical Area (1992-2000). "? limited to participants who did not change primarily residential address between 1976 and 1992. ${ }^{+}$: models are for 1992-2000 and for a $10-\mu \mathrm{g} \cdot \mathrm{m}^{-3}$ increase in 4-year moving average particulate matter exposures. ${ }^{\S}$ : models are for $1992-2000$ and for each $10-\mu \mathrm{g} \cdot \mathrm{m}^{-3}$ increase in average particulate matter exposures; there were 104254 women included in the asthma analysis and 103838 women included in the chronic obstructive pulmonary disease analysis. ${ }^{f}$ : models adjusted for age, time period and geographic region. \#\#: additionally adjusted for body mass index, alcohol consumption, physical activity, census-tract median household income, Western dietary pattern [12], second-hand smoke exposure at home and work, smoking status (when not stratified by status), and pack-years. 
The spatiotemporal models also did not account for differences in time-activity patterns, time spent outdoors or time spent at the residence. Although mechanisms of action may take decades of exposure, we were limited to a shorter follow-up period due to the availability of pollution data and the date of the last supplemental asthma/COPD questionnaire. Although we used validated case definitions of a self-reported physician diagnosis, we could not exclude the possibility of undiagnosed or unreported disease. Additionally, some incident cases of asthma may represent forgotten or undiagnosed reactivations from earlier in life, a limitation shared by most studies of adult-onset asthma. Generalisability of these findings outside of this cohort of predominantly white nurses may be limited. In conclusion, we found no evidence that long-term exposure to particulate matter increased the risk of incident asthma or COPD in this cohort.

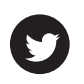

@ERSpublications

In the NHS cohort, lack of strong evidence for long-term PM association with adult-onset asthma and COPD http://ow.ly/960A300pUSA

\begin{abstract}
Jared A. Fisher ${ }^{1,2}$, Robin C. Puett ${ }^{1,2}$, Jaime E. Hart ${ }^{3,4}$, Carlos A. Camargo Jr ${ }^{3,5,6}$, Raphaëlle Varraso ${ }^{7,8}$, Jeff D. Yanosky ${ }^{9}$ and Francine Laden ${ }^{3,4,6}$

${ }^{1}$ Maryland Institute for Applied Environmental Health, University of Maryland School of Public Health, College Park, MD, USA. ${ }^{2}$ Dept of Epidemiology and Biostatistics, University of Maryland School of Public Health, College Park, MD, USA. ${ }^{3}$ Channing Division of Network Medicine, Dept of Medicine, Brigham and Women's Hospital and Harvard Medical School, Boston, MA, USA. ${ }^{4}$ Dept of Environmental Health, Harvard T.H. Chan School of Public Health, Boston, MA, USA. ${ }^{5}$ Dept of Emergency Medicine, Massachusetts General Hospital, Harvard Medical School, Boston, MA, USA. ${ }^{6}$ Dept of Epidemiology, Harvard T.H. Chan School of Public Health, Boston, MA, USA. ${ }^{7}$ INSERM U1168, VIMA (Aging and Chronic Diseases: Epidemiological and Public Health Approaches), Villejuif, France. ${ }^{8}$ UMR-S 1168, Université Versailles St-Quentin-en-Yvelines, Versailles, France. ${ }^{9}$ Dept of Public Health Sciences, Pennsylvania State University College of Medicine, Hershey, PA, USA.
\end{abstract}

Correspondence: Jared Fisher, University of Maryland School of Public Health, 255 Valley Drive, Room 1222, College Park, MD 20742, USA. E-mail: jafisher@umd.edu

Received: May 282015 | Accepted after revision: May 092016 | First published online: June 102016

Support statement: Financial support was provided by the US National Institutes of Health (grants R01ES017017 and UM1 CA186107). Funding information for this article has been deposited with the Open Funder Registry.

Conflict of interest: Disclosures can be found alongside this article at erj.ersjournals.com

Acknowledgements: We thank the participants and staff of the Nurses' Health Study for their valuable contributions.

\title{
References
}

1 Brunekreef B, Holgate ST. Air pollution and health. Lancet 2002; 360: 1233-1242.

2 Zanobetti A, Bind MA, Schwartz J. Particulate air pollution and survival in a COPD cohort. Environ Health 2008; 7: 48 .

3 Jacquemin B, Schikowski T, Carsin AE, et al. The role of air pollution in adult-onset asthma: a review of the current evidence. Semin Respir Crit Care Med 2012; 33: 606-619.

4 Schikowski T, Mills IC, Anderson HR, et al. Ambient air pollution: a cause of COPD?. Eur Respir J 2014; 43: 250-263.

5 Young MT, Sandler DP, DeRoo LA, et al. Ambient air pollution exposure and incident adult asthma in a nationwide cohort of U.S. women. Am J Respir Crit Care Med 2014; 190: 914-921.

6 Schikowski T, Adam M, Marcon A, et al. Association of ambient air pollution with the prevalence and incidence of COPD. Eur Respir J 2014; 44: 614-626.

7 Jacquemin B, Siroux V, Sanchez M, et al. Ambient Air Pollution and Adult Asthma Incidence in Six European Cohorts (ESCAPE). Environ Health Perspect 2015; 123: 613-621.

8 Yanosky JD, Paciorek CJ, Laden F, et al. Spatio-temporal modeling of particulate air pollution in the conterminous United States using geographic and meteorological predictors. Environ Health 2014; 13: 63.

9 Camargo CA Jr, Weiss ST, Zhang S, et al. Prospective study of body mass index, weight change, and risk of adult-onset asthma in women. Arch Intern Med 1999; 159: 2582-2588.

10 Barr RG, Herbstman J, Speizer FE Jr, et al. Validation of self-reported chronic obstructive pulmonary disease in a cohort study of nurses. Am J Epidemiol 2002; 155: 965-971.

11 US Census Bureau. Census 2000 TIGER/Line ${ }^{\circ}$ Files: Technical Documentation. www2.census.gov/geo/pdfs/ maps-data/data/tiger/rd_2ktiger/tgrrd2k.pdf Date last accessed: May 21, 2015. Date last updated: 2001.

12 Varraso R, Fung TT, Barr RG, et al. Prospective study of dietary patterns and chronic obstructive pulmonary disease among US women. Am J Clin Nutr 2007; 86: 488-495.

13 Gowers AM, Cullinan P, Ayres JG, et al. Does outdoor air pollution induce new cases of asthma? Biological plausibility and evidence; a review. Respirology 2012; 17: 887-898.

14 Grievink L, Smit HA, Brunekreef B. Anti-oxidants and air pollution in relation to indicators of asthma and COPD: a review of the current evidence. Clin Exp Allergy 2000; 30: 1344-1354.

15 Zuo L, He F, Sergakis GG, et al. Interrelated role of cigarette smoking, oxidative stress, and immune response in COPD and corresponding treatments. Am J Physiol Lung Cell Mol Physiol 2014; 307: L205-L218. 